\title{
Muséologies
}

Les cahiers d'études supérieures

\section{De la structure au devenir : les récits d'Europunk}

\section{Hélène Laurin}

Volume 8, numéro 1, 2015

Réflexions sur la pratique curatoriale et la recherche-création

URI : https://id.erudit.org/iderudit/1034617ar

DOI : https://doi.org/10.7202/1034617ar

Aller au sommaire du numéro

Éditeur(s)

Association Québécoise de Promotion des Recherches Étudiantes en

Muséologie (AQPREM)

ISSN

1718-5181 (imprimé)

1929-7815 (numérique)

Découvrir la revue

Citer ce compte rendu

Laurin, H. (2015). Compte rendu de [De la structure au devenir : les récits d’Europunk]. Muséologies, 8(1), 191-207. https://doi.org/10.7202/1034617ar

Tous droits réservés (C) Association Québécoise de Promotion des Recherches Étudiantes en Muséologie (AQPREM), 2016
Ce document est protégé par la loi sur le droit d'auteur. L'utilisation des services d'Érudit (y compris la reproduction) est assujettie à sa politique d'utilisation que vous pouvez consulter en ligne.

https://apropos.erudit.org/fr/usagers/politique-dutilisation/ 
Carnet trois

De la structure au devenir : les récits d'Europunk

Hélène Laurin 
Titulaire d'un doctorat en études de la communication de l'Université McGill, Hélène Laurin a été boursière postdoctorale du Fonds de recherche du Québec Société et Culture (FRQSC) à l'École d'études politiques de l'Université d'Ottawa. Elle s'intéresse particulièrement aux manières dont les personnes, les choses et les événements gagnent et perdent socialement de la valeur. laurinh@videotron.ca 
Les résultats des recherches effectuées par les musées doivent être articulés et mis en forme d'une façon ou d'une autre dans le but d'en faire une exposition. Le processus muséal présuppose en effet que la recherche doit être traduite dans une ou des salles, en trois dimensions, ce qui est loin d'être évident. Une des manières d'y arriver est de traduire les résultats de recherche en récit. De fait, le récit est une forme particulièrement polymorphe qui se prête bien à l'exercice créatif de l'adaptation de la recherche, nécessaire en vue de la rendre accessible et abordable tout en conservant la complexité du phénomène étudié. De plus, les œuvres d'art et les artéfacts mis en valeur dans les expositions muséales sont eux-mêmes traversés de récits: les récits de leur fabrication, les récits de leur circulation, les récits de leur interprétation, entre autres.

La présente contribution consiste en une lecture conceptuelle du récit formé par la mise en exposition; en ce sens, elle est personnelle. J'aborderai le récit selon trois apports narratologiques: la conception structuraliste, la conception performative et la conception ontogénétique. Pour illustrer celles-ci, ainsi que les différentes facettes mises de l'avant par chaque conception, j'utiliserai le contenu d'une seule exposition, Europunk, exposition temporaire développée par l'Académie de France à Rome - Villa Médicis et présentée dans une forme renouvelée à la Cité de la musique à Paris' ${ }^{1}$ à la fin de 2013.

\section{Le mouvement punk et Europunk}

Le punk consiste en une musique et une culture en soi. Puisque plusieurs récits concurrents du punk coexistent, voici la version la plus consensuelle et hégémonique de ce mouvement. Le punk s'est développé au milieu des années 1970, surtout en Grande-Bretagne et aux États-Unis. Des groupes de musique sont alors apparus, présentant une musique abrasive et minimale, avec des paroles souvent politiques, versant surtout dans le cynisme, mais parfois personnelles, poétiques ou même festives. Parmi les groupes influents, notons The Sex Pistols, The Clash, The Ramones, The Germs, Siouxsie and the Banshees, et Patti Smith. Vers le début des années 1980, une branche du punk s'est mutée en musique dite new wave, avec un son intégrant claviers et rythmes sautillants, gagnant ainsi en popularité.

Au point de vue culturel, le mouvement punk privilégie tout ce qui se pose contre l'ordre établi: les punks dérangent, ils sont antiautoritaires et ils ont développé une éthique autosuffisante où musiques, fanzines, vêtements et autres biens sont créés par les individus concernés, sans aide extérieure (une éthique aussi appelée do-it-yourself ou simplement

1 La Cité de la musique a depuis été rénovée et s'appelle maintenant la Philharmonie de Paris. Puisque Europunk prenait place dans "l'ancienne " Cité de la musique, je continuerai à faire référence à celle-ci plutôt qu'à la Philharmonie de Paris.
En outre, les photos qui apparaissent dans cet article proviennent de la version originale de l'exposition à l'Académie de France à Rome - Villa Médicis, que je souhaite remercier d'ailleurs de leur collaboration. 
DIY). Conséquemment, le punk est aussi un mouvement grassroots, au sens où la prise de parole, comprise au sens large (composition de chansons, constitution d'un groupe de musique, réalisation d'un fanzine, création d'une œuvre d'art), y est encouragée pour tous. Le punk a touché d'autres formes artistiques que la musique, toujours selon ces mêmes préceptes; parmi celles-ci, notons la mode, la littérature, l'art vidéo et les arts visuels. C'est ici que l'exposition Europunk intervient.

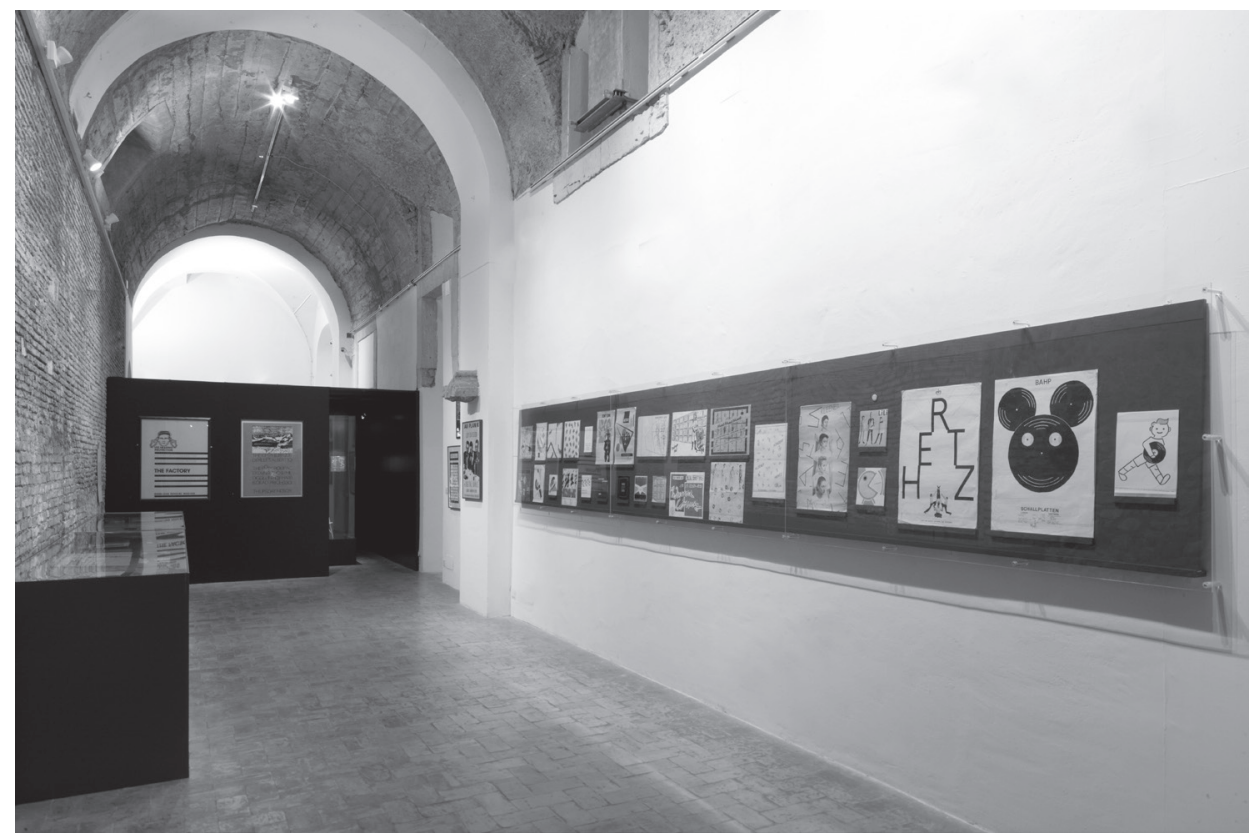

Europunk la culture visuelle punk en Europe, 1976-1980.

[Photo: Giovanni De Angelis]. (c) Giovanni De Angelis, photographe, Académie de France à Rome - Villa Médicis].

Cette exposition porte spécifiquement sur la créativité visuelle de la culture punk en Europe entre 1976 et 1980. Près de 500 artéfacts associés au punk européen (affiches, fanzines, brochures, pochettes de disque, tracts et pièces de vêtement) sont répartis en six sections: Sex Pistols, Bazooka (un collectif visuel français d'allégeance punk), WTF, Anarchy, DIY et New Wave. Ces sections comportent des éléments, descriptifs ou historiques, qui sont devenus synonymes de la culture punk; chacune est introduite par des explications minimales et elles ne sont que très peu reliées entre elles².

En outre, en plein centre de la salle où a lieu Europunk se déroule une double chronologie. Cette dernière est affichée sur dix murets s'enfilant les uns derrière les autres, un peu comme des dominos. Chaque

2 Cela diffère de plusieurs expositions muséales portant sur des phénomènes musicaux, habituellement narrativisées de manière à enseigner l'histoire ou l'importance d'un courant ou d'un artiste (par exemple David Bowie Is, développée et présentée par le Victoria and Albert Museum en 2013). J'ai choisi Europunk justement à cause de son aspect atypique, caractéristique qui fait de l'exposition un cas limite et rend son analyse particulièrement stimulante. 
muret représente une année, de 1970 à 1980. Sur les côtés faisant face à la porte d'entrée de l'exposition sont exposés les événements relatifs au punk de l'année représentée; sur le « verso " des murets figurent les événements de l'actualité de la même année, des événements économiques, politiques et sociaux (d'où le nom que je donne à cet arrangement: « double » chronologie). Les événements, tant ceux relatifs au punk que ceux d'actualité, sont résumés en une phrase ou deux et sont ordonnés selon leur date. Quelques écrans de télévision diffusent en boucle des images des événements en question. Le son est coupé, mais des écouteurs sont disponibles pour qui veut entendre ce qui est montré sur les téléviseurs. II m'a semblé que les événements d'actualité, attestant du chaos et des temps difficiles de la décennie 1970 (crise du pétrole, terrorisme nationaliste en Italie et en Allemagne, marées noires, et bien d'autres), ont été choisis afin d'établir un lien avec la montée du punk, une musique difficile et nihiliste, comme si les uns étaient un reflet de l'autre.

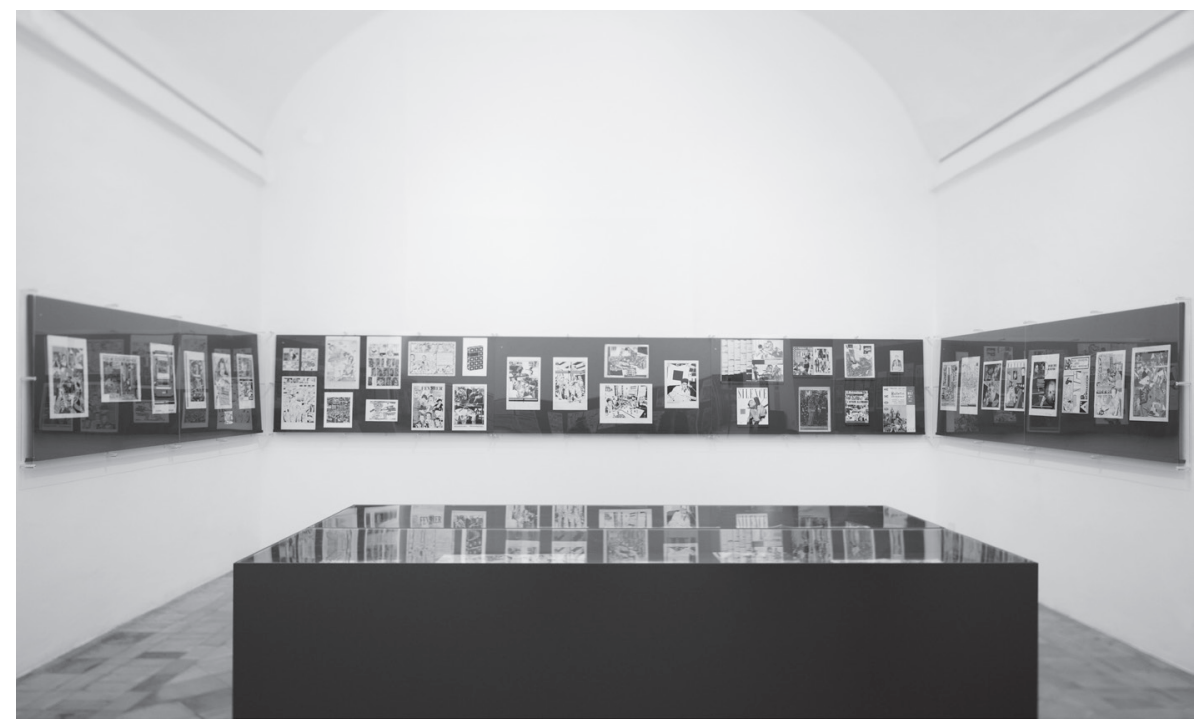

Europunk la culture visuelle punk en Europe, 1976-1980.

[Photo: Giovanni De Angelis]. (C) Giovanni De Angelis, photographe, Académie de France à Rome - Villa Médicis].

Enfin, l'exposition comprend une petite salle, séparée mais adjacente à la salle d'exposition principale, où il est possible d'apprendre les rudiments de la musique punk sur quelques instruments. Cet atelier en accès libre, appelé Play it, met en effet à disposition des visiteurs adolescents et adultes une guitare électrique, une basse électrique, un clavier et une batterie. Un musicien-médiateur est sur place pour conseiller les musiciens en herbe. Clairement conçu pour insuffler une dose éducative et interactive à l'exposition, en plus de correspondre aux valeurs do-it-yourself du punk, cet atelier m'a semblé très populaire au moment de ma visite, vu la longueur considérable de la file d'attente à la porte. En somme, « l'exposition Europunk redonne un crédit artistique à toute une culture visuelle spécifique qui s'est pourtant bâtie en 
totale opposition à l'art ${ }^{3}$ ». Comme déjà soulevé, le récit du mouvement punk a été si souvent raconté qu'il est maintenant " cristallisé "; il existe donc un point de comparaison assez fort à propos du punk, contre et avec lequel ce qui est raconté dans Europunk se frotte. Les lectures conceptuelles du récit formé par la mise en exposition proposées dans cet article seront donc teintées de cette comparaison.

\section{Récits structuralistes}

Tout d'abord, le courant structuraliste, qui a connu son apogée entre 1950 et 1970, provient de la distinction entre parole et langue établie par le linguiste suisse Ferdinand de Saussure (1857-1973). Alors que la parole comporte les différentes itérations individuelles, la langue constitue l'ensemble des règles et des relations du langage, c'est-àdire sa structure ${ }^{4}$. Les structuralistes font le pari que ce genre d'opposition se retrouve à la base même de nombreux phénomènes sociaux et de formes culturelles, et le récit fait justement partie de ceux-ci. Parmi les conceptions structuralistes du récit qui ont été particulièrement influentes, notons celle du formaliste russe Vladimir Propp ${ }^{5}$. Dans son étude Morphologie du conte, publiée originalement en 1928, il a dégagé 31 fonctions, soit des unités narratives, formant la structure fondamentale de contes traditionnels russes. Grandement influencé par Propp, le sémioticien Algirdas Julien Greimas a conçu un schéma actantiel formé de six composantes ${ }^{6}$. Ces fonctions et ces catégories de personnages constituent des structures permettant à la fois d'engendrer, d'établir et d'analyser les manières dont les récits se déploient, et elles peuvent être appliquées à pratiquement n'importe quel récit, en littérature, cinéma, théâtre, télévision, jeu vidéo, etc.

La division langue/parole ainsi que les différents modèles du récit qui en ont émergé sous-entendent un binôme important pour la suite des choses: I'histoire et le discours. Dans un premier temps, l'histoire, soit le contenu du récit, consiste en les événements, le quoi du récit? ${ }^{7}$. Dans un deuxième temps, le discours est l'ensemble des manières par lesquelles l'histoire est racontée, le comment du récit ${ }^{8}$. Ces deux éléments ont porté plusieurs noms: le fabula et le sjuzet des formalistes russes, le narrated et le narrating, le contenu et l'expression, la structure profonde et la manifestation de surface, notamment. Autrement dit, les narratologistes d'allégeance structuraliste posent l'existence de deux niveaux indépendants constituant le récit: l'histoire

3 CITÉ DE LA MUSIQUE. Exposition Europunk. 15 octobre 2013 - 19 janvier 2014.

4 ATTALAH, Paul. Théories de la communication: sens, sujets, savoirs. Québec: Presses de l’Université du Québec, 1991, p. 268-270.

5 PROPP, Vladimir. Morphologie du conte. Paris:

Gallimard, 1970.

6 Le héros se fait donner une mission par un émetteur pour

trouver ou recouvrer un objet - ou un objectif - qui sera au

bénéfice d'un récepteur. Le héros, dans sa quête (celle-ci est parfois considérée comme une composante du schéma actantiel), sera aidé par un adjuvant et ses plans seront contrecarrés par un opposant. Voir GREIMAS, Algirdas Julien. «Éléments pour une théorie de l'interprétation du récit mythique ". Communications, vol. 8, $\mathrm{n}^{\circ} 1,1966$, p. 28-59.

7 O'NEILL, Patrick. Fictions of Discourse: Reading Narrative Theory. Toronto et Buffalo: University of Toronto Press, 1996, p. 3.

8 Ibid. 
de base, isolable et inchangeante, qui est renouvelée à chaque fois par le niveau supérieur, celui du discours ${ }^{9}$.

Plusieurs arguments soutiennent cette perspective. Celui qui est le plus souvent invoqué est le fait que I'histoire soit transposable, en ce sens que la même histoire existe selon plusieurs versions différentes et selon plusieurs modes et médias; en somme, une histoire se transpose selon plusieurs discours ${ }^{10}$. Ce potentiel de métamorphose est partout présent dans nos vies quotidiennes: pensons à « l'adaptation cinématographique " d'un roman, à la « version abrégée " d'un classique de la littérature, à la « version Disney " d'un conte, à la « version des faits » d'un témoin important, et ainsi de suite. La temporalité du récit est à la source de deux autres arguments fréquemment employés pour justifier le dédoublement entre histoire et discours. Tout d'abord, la différence entre le temps du discours et le temps de l'histoire; un écart subsiste entre la plage de temps au cours de laquelle les événements racontés se déroulent - ce qui constitue l'histoire - et le temps nécessaire pour lire, raconter ou entendre un récit - le discours ${ }^{11}$. Ensuite, un ensemble d'événements qui se déroulent dans un ordre particulier peut être narré dans un autre ordre, tout en gardant son unité et sa cohérence ${ }^{12}$.

En adoptant la perspective structuraliste pour analyser le récit de l'exposition Europunk, on se rend compte que cette exposition montre une version de l'histoire de base du punk, une histoire qui est à maints égards plutôt figée dans une forme dont on reconnaît sans peine les contours. Par exemple, les six sections de l'exposition résument très bien la teneur du mouvement punk. Et il est possible de comparer la version de l'exposition avec d'autres versions et ainsi prouver leur similarité. Les Sex Pistols, à qui est consacrée la première section dans Europunk, symbolisent le punk, selon leur entrée dans le Dictionnaire du rock: « ce groupe londonien a résumé, par son parcours fulgurant, un mouvement artistique révolutionnaire: le punk-rock ${ }^{13}$ ». Pareillement, l'idée du punk comme synonyme d'anarchie et d'art do-it-yourself, là aussi deux sections d'Europunk, est si répandue et connue qu'elle inspire même une auteure du blogue de l'empire Martha Stewart, extrême représentant de l'ordre établi s'il en est un, d'en faire le thème d'une fête pour enfants. Cette chroniqueuse résume le courant punk ainsi: " since it hit the mainstream around the 1970s, punk as a subculture has enamored kids (of all ages) with its attitude of freedom, self-expression, and do-it-yourself creativity ${ }^{14}$ ". D'ailleurs, dans Europunk, la parcimonie des textes, comparée à la prolifération des artéfacts, met encore plus en lumière l'importance de l'histoire

9 HERRNSTEIN SMITH, Barbara. " Narrative Versions, Narrative Theories ". Critical Inquiry, vol. 7, n 1, 1980, p. 213-216.

10 CHATMAN, Seymour. Story and Discourse: Narrative Structure in Fiction and Film. Ithaca: Cornell University Press, 1978, p. 20.

11 Id., p. 62-63.

12 GOODMAN, Nelson. "Twisted Tales ». Critical Inquiry, vol. $7, \mathrm{n}^{\circ} 1,1980$, p. 104 .
13 CARON, François. "Sex Pistols ». In. ASSAYAS, Michka (dir.). Dictionnaire du rock. Paris : Robert Laffont, 2000, p. 1695.

14 STEWART, Martha. Rock On! How to Throw a Punk Rock-Inspired Party. <http://www.marthastewart. com/1085315/party-themes-punk-rock> (consulté le 24 octobre 2014) 
de base du punk dans l'exposition, puisque doit y être distillée, en quelques phrases, toute la teneur de chacune des sections ${ }^{15}$.

Europunk se lit également d'une manière structuraliste par le déploiement du modèle habituel de l'exposition muséale. En effet, non seulement les récits peuvent être décodés en fonction du structuralisme, mais les différentes formes culturelles, comme la mise en exposition d'un phénomène, peuvent l'être aussi. En ce sens, l'exposition est lisible selon un " modèle générateur d'exposition muséale », une sorte d'équivalent du schéma actantiel vu précédemment, adapté aux musées. L'exposition contient effectivement une mise en contexte, des textes, des artéfacts et un parcours relativement prédéfini, éléments non seulement présents dans plusieurs expositions (à propos de la musique ou de n'importe quel autre sujet), mais qui en outre " engendrent »l'exposition telle que les visiteurs l'expérimentent.

L'angle structuraliste $n$ 'est cependant pas sans faille, particulièrement quand vient le temps de considérer un récit d'exposition. En premier lieu, revenons sur la question du dédoublement entre histoire et discours. En quoi, précisément, consiste cette histoire de base? Est-il réellement possible de tirer un substrat du récit, indépendant de I'instance qui raconte, de ses intérêts, de sa mémoire, de ses choix, de sa perception et de ses interactions? S'il est possible de distinguer des contours semblables dans plusieurs versions d'un même récit, comme il a été possible de le faire dans le cas du punk et d'Europunk, cela est peut-être davantage dû à la circulation de versions (d'une version?) hégémoniques du récit de l'histoire du punk, où certains éléments - comme l'importance des Sex Pistols, l'aspect DIY - ont été favorisés par rapport à d'autres. Autrement dit, I'histoire de base d'un récit révèle peut-être moins une " essence » du récit que l'homogénéité des manières dont plusieurs individus lisent, ou plus largement reçoivent, ce récit. Pour les musées, pendant la période préparatoire, la conception structuraliste peut certainement aider à concevoir l'exposition, puisque cette théorie comporte un aspect systématique facilitant l'opérationnalisation de la mise en exposition. Cependant, le danger est de croire que le récit présenté est une histoire de base, une sorte de version idéale, pure, sans apports extérieurs et sans impact sur les versions subséquentes du récit ${ }^{16}$.

En deuxième lieu, il y a un enjeu lié à la durée et à l'ordre changeants entre l'histoire et le discours, caractéristiques démontrant le bien-fondé du structuralisme, tel que vu plus haut. Pour reprendre Europunk, qui couvre les cinq dernières années de la décennie 1970, cela prend évidemment beaucoup moins de temps de parcourir l'exposition que de vivre ces cinq années; également, dans le récit d'Europunk, l'ordre de ces cinq années n'est pas chronologique, puisque le récit fonctionne

15 Pour donner une idée des textes de l'exposition, voir le site de la CITÉ DE LA MUSIQUE. Europunk. <http://www. citedelamusique.fr/minisites/1310_europunk/index.asp> (consulté le 6 novembre 2014).
16 Je ne veux pas suggérer ici que le commissaire d'Europunk soit tombé dans ce panneau; comme mentionné, j'utilise cette exposition comme exemple pour illustrer tour à tour trois théories aidant à concevoir les récits de recherches. 
par thème. Cela mentionné, cette différence résulte de l'aspect conventionné du langage: " there may be and usually are differences between the formal properties of a linguistic event and the set of conditions conventionally implied by the occurrence of an event of that form ${ }^{17}$ ». Pourquoi la correspondance entre l'ordre et la durée de l'histoire et du discours serait-elle alors si importante, si cruciale? Pourquoi mettre l'accent sur cette différence pour valider le structuralisme? La réponse réside dans la question: à cause de l'importance donnée à la division entre histoire et discours, qui est au cœur même de la conception structuraliste du récit.

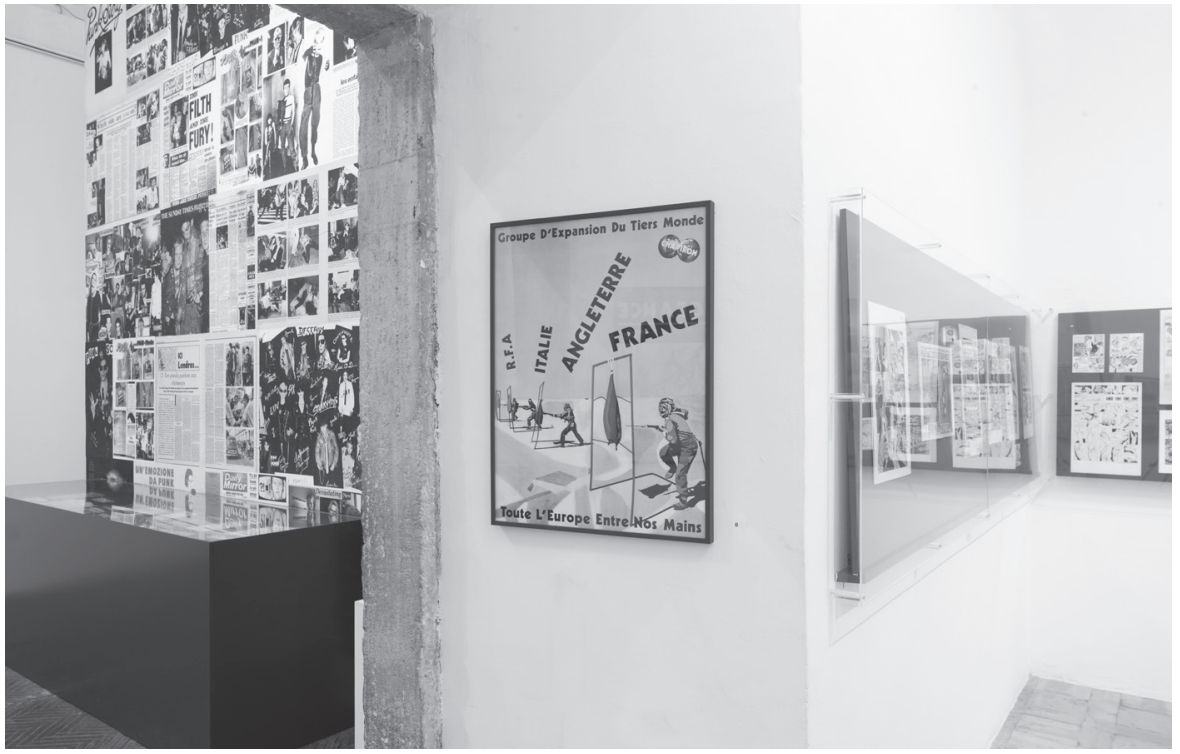

Europunk la culture visuelle punk en Europe, 1976-1980.

[Photo: Giovanni De Angelis]. (C) Giovanni De Angelis, photographe, Académie de France à Rome - Villa Médicis].

En troisième lieu, et ce point poursuit le questionnement du paragraphe précédent, la conception structuraliste du récit suppose une forme très spécifique. Or, un récit ne se présente pas toujours sous la forme « il était une fois ». Par exemple, un concept issu de la biologie, en apparence difficile à saisir, peut être vulgarisé à l'aide d'une comparaison comportant une part narrative; la dimension communicative ainsi évoquée par la comparaison tendrait à dissoudre tous types de frontières strictes au récit ${ }^{18}$. En d'autres mots, les manifestations du récit dépassent largement le modèle du conte qui est à la base de la conceptualisation structuraliste. Les récits fonctionnent avec des dimensions qui ne relèvent pas nécessairement du récit. Ils ne sont pas faits de pièces détachables et rattachables à souhait, ils sont plutôt

17 HERRNSTEIN SMITH, « Narrative Versions, Narrative Theories ", op. cit., p. 224.
18 CAMBRON, Micheline. Une société, un récit: discours culturel au Québec, 1967-1976. Montréal: L’Hexagone, 1989, p. 15. 
inscrits dans un processus. Et il est possible de voir Europunk ainsi: non seulement le récit, divisé en six thèmes, peut être considéré comme ténu, mais il fonctionne surtout avec des éléments non narratifs, comme les artéfacts qui y sont présentés en très grand nombre. En conséquence, il serait profitable d'envisager les recherches traduites en récit d'exposition comme une transaction, comme un acte qui accomplit quelque chose.

\section{Récits performatifs}

Le philosophe britannique John L. Austin, avec sa suite de conférences publiées dans Quand dire, c'est faire ${ }^{19}$, a été parmi les premiers à élaborer la théorie des actes de langage, qui fait partie de la grande famille des théories de la performativité. En quelques mots, Austin a analysé les manières dont certains énoncés ne sont ni vrais, ni faux, mais " accomplissent " plutôt des actions. Suivant les circonstances, ces énoncés dits performatifs se soldent par des succès ou des insuccès, c'est-à-dire selon que tout est en place pour qu'ils soient compris, acceptés et performés. Ainsi, une panoplie d'actions sont réalisées par l'intermédiaire des paroles: excuses, promesses, offres, paris, affirmations, invitations, ordres, etc. Au sens plus large, la théorie des actes de langage peut être pensée en conjonction avec les récits. Ceux-ci, vus selon la perspective performative, consistent en des réactions produites avec et contre de nombreuses conditions. Ces conditions forment la conjoncture du récit, cette grande oubliée du structuralisme.

En suivant la performativité, les différentes versions d'un même récit se moulent non pas à l'aune d'une histoire de base, mais en fonction de deux grandes catégories de conditions. Barbara Herrnstein Smith les divise en variables circonstancielles et en variables psychologiques ${ }^{20}$. Les premières font référence à la conjoncture culturelle, sociale et matérielle selon laquelle un récit est façonné, raconté et compris: pensons notamment à l'environnement physique, à la nature des récepteurs (ceux qui écoutent ou lisent le récit) et à la relation entre ceux-ci et le narrateur ${ }^{21}$. Les secondes conditions se rapportent justement au narrateur: ses motivations, ses intérêts, ses désirs, ses attentes, sa mémoire, sa connaissance des événements, mais aussi son capital culturel des conventions et des traditions ayant trait à la narration, et enfin son capital symbolique, qui le pousse à raconter un récit choisi, à un public donné, à une occasion particulière et $d^{\prime}$ une manière précise, façonnant ainsi sa réception ${ }^{22}$. En bout de compte, c'est le lecteur qui interprète le récit; c'est donc lui qui, après tout, agit: selon la théorie performative, un récit pourrait se définir comme " a perceived sequence of non-randomly connected events ${ }^{23} »$. Il est donc davantage question

19 AUSTIN, John L. Quand dire, c'est faire [Trad. Gilles Lane]. Paris: Seuil, 1970.

20 HERRNSTEIN SMITH, « Narrative Versions, Narrative Theories ", op. cit., p. 225-226.

21 Ibid. 1988, p. 7. 
de fonctions du récit que de propriétés formelles, comme c'est le cas selon la théorie structuraliste. À l'instar des paroles, les récits agissent en promettant, ordonnant, classant, validant, et bien plus.

Cela relève de la question du pouvoir. Selon la théorie performative, les récits exercent une sorte de pouvoir, un pouvoir qui est souvent sublimé ou naturalisé, donc oublié. Comme mentionné précédemment, le capital symbolique du narrateur et celui du récepteur font partie intégrante de la conjoncture permettant à un récit d'accomplir quelque chose en particulier. Autrement dit, si une personne ou une institution déploie correctement les conventions reliées à la narration d'un récit particulier, et si cette personne ou institution est reconnue comme possédant du pouvoir, l'impact de ses mots peut être considérable ${ }^{24}$. À cet égard, le récit est vu comme un " instrument of the powerful ideologies played out in cultural practices ${ }^{25} "$, au confluent de plusieurs forces culturelles, sociales et idéologiques. Les récits constituent donc, selon la théorie performative, des vecteurs permettant la circulation et la compréhension, à grande ou à petite échelle, d'événements, de personnages, de lois (tacites ou admises), d'expériences partagées, de mémoires collectives, de phénomènes sociaux.

La lecture du récit articulé dans l'exposition Europunk selon la théorie performative doit impérativement prendre en compte la conjoncture muséale dans laquelle elle s'insère. La Cité de la musique est un établissement entièrement dédié à la musique et comportant salle de spectacle, médiathèque, librairie et musée. Le Musée de la musique s'enorgueillit de sa collection composée de 4000 instruments et de sa programmation muséale "riche et diversifiée ${ }^{26}$ ", selon les mots même de Laurent Bayle, directeur général de la Cité de la musique, et d'Éric de Visscher, directeur du Musée de la musique. Un simple coup d'œil à la programmation passée confirme cette hétérogénéité: de Jimi Hendrix à la musique indienne, de la musique du Moyen Âge à Bob Dylan, de la musique populaire brésilienne à Miles Davis, etc. D'ailleurs, le Musée de la musique articule grandement sa pertinence sociale et culturelle avec ces expositions temporaires - l'institution en organise en moyenne trois par année depuis 1999. Enfin, la librairie, qui pourrait en outre être considérée comme une boutique de souvenirs, concentre son offre surtout autour de la musique classique ${ }^{27}$ et du contenu de l'exposition temporaire du moment. Europunk et son récit participent à cette conjoncture.

Deux éléments apparaissent déterminants pour la lecture de la performativité du récit articulé dans Europunk: tout d'abord, le punk est une culture musicale reconnue comme anti-establishment et la voici

24 BOURDIEU, Pierre. Langage et pouvoir symbolique. Paris: Seuil, 2001, p. 99-131.

25 CARLISLE, Janice. «Introduction ». In. CARLISLE, Janice et Daniel R. SCHWARZ (dir.). Narrative and Culture. Athens: University of Georgia Press, p. 10.
26 BAYLE, Laurent et Éric DE VISSCHER. « Préface ». In. Europunk: Une révolution artistique en Europe [1976-1980]. Paris: Cité de la musique, 2013, p. 7.

27 La Philharmonie de Paris a résidence à la Cité de la musique et la programmation de la Salle Pleyel, faisant partie de la Cité également, est largement axée sur la musique classique. 
figée dans un musée; ensuite, l'exposition, sise au Musée de la musique, insiste pourtant sur l'aspect visuel du punk. Passons en revue ces éléments. Premièrement, tel que mentionné - et il s'agit là d'un fait assez connu et consensuel -, la culture punk a été un mouvement se caractérisant par son refus de l'ordre et de l'autorité. Cela s'est incarné jusque dans les pratiques artistiques visuelles montrées dans Europunk: n'importe qui pouvait prendre des crayons, des ciseaux et de la colle et agencer un fanzine distribué de main à main à un groupe d'amis ou dans une salle de spectacle improvisée. Ce n'est pas un mouvement qui, à sa base, voulait se retrouver dans un musée, bien au contraire. Comme le mentionnent Bayle et de Visscher dans la préface du catalogue de l'exposition, "Que le punk entre au musée n'est pas le moindre des paradoxes: mis à part Dada dont il peut être considéré comme un héritier lointain, existe-t-il un courant artistique plus iconoclaste, plus anti-art (et anti-musique) que ce cri de révolte $[\ldots]^{28}$ ? " Le musée, comme institution, incarne un pouvoir difficile à nier. Et même si Bayle, de Visscher et même le commissaire de l'exposition Éric de Chassey reconnaissent les positions antagonistes partagées par le punk et le musée ${ }^{29}$, tous s'accordent pour affirmer haut et fort la pertinence et la justesse d'avoir une exposition muséale consacrée au punk:

Que la Cité de la musique s'intéresse au punk n'est pas un hasard: en reprenant et en adaptant l'exposition conçue par Éric de Chassey pour la Villa Médicis à Rome, le Musée de la musique rend hommage au rôle pionnier - et parfois excessif - de ces créateurs qui affirmaient que la musique se créait avec trois accords et que l'art pouvait se contenter de la reproduction de clichés empruntés à l'imagerie du quotidien ${ }^{30}$.

De Chassey reprend ce fil, en insistant sur un autre point: " une trentaine d'années après les faits, alors que l'influence du mouvement ne cesse de se faire sentir dans tous les domaines de la création actuelle, autant en termes de formes que de méthodes, cela [la muséification] devient nécessaire ${ }^{31}$ ». En somme, le Musée de la musique et ses délégués que constituent Bayle, de Visscher et de Chassey exercent leur pouvoir, dans une perspective performative, en affirmant rendre hommage au mouvement punk et en reconnaissant son influence.

Deuxièmement, la performativité apparaît dans la présence d'œuvres visuelles dans un musée dédié à la musique. Le récit d'Europunk comprend près de 500 artéfacts, dans un espace qui n'est pas si vaste. C'est suffisant pour noyer le visiteur, pour saturer son champ de vision. Aussi, et de manière importante, de Chassey explique

28 BAYLE et DE VISSCHER, "Préface ", op. cit., p. 7.

29 Dans l'introduction au catalogue, de Chassey écrit: «Il aurait sans doute été mortifère de rendre compte trop tôt de la dimension proprement artistique de cette ambition de changer le monde [du mouvement punk], sans réduire à néant la validité de l'opposition frontale à la muséification. " DE CHASSEY, Éric. «Europunk ». In. Europunk: une révolution artistique en Europe [1976-1980]. Paris: Cité de la musique, 2013, p. 9.
30 BAYLE et DE VISSCHER, "Préface ", op. cit., p. 7.

31 DE CHASSEY, « Europunk », op. cit., p. 9. 
dans le programme gratuit de l'exposition comment considérer ces objets: "Ce n'est pas leur dimension anecdotique qui m'intéresse dans ces objets. Le principe premier de l'exposition est justement de ne pas considérer ces objets comme des documents, mais comme des œuvres ${ }^{32}$. „ Ces œuvres d'art visuel sont donc entrées dans un musée habituellement consacré à la musique. Ajoutant à la saturation visuelle, très peu d'entre elles sont commentées, elles ne sont qu'affichées. D'ailleurs, avant d'entrer dans la salle de l'exposition, le visiteur doit passer par la librairie, très axée sur la musique classique. II y a déjà un sentiment de révérence déployé avant même l'entrée de la salle d'exposition d'Europunk, institué par le capital symbolique muséal, mais aussi par la disposition architecturale même de la Cité de la musique. De cette manière, l'intention derrière le récit d'Europunk se devine même si le catalogue ou le programme n'a pas été lu au préalable: celle de modifier le point de vue consensuel par rapport au punk, l'animer d'un souffle artistique valide. Selon la performativité, la mise en récit d'Europunk produit donc quelque chose; dans ce cas-ci, elle produit une légitimation et une nouvelle interprétation du punk, une interprétation basée sur la qualité artistique du mouvement, prenant la créativité visuelle comme preuve. Pour la recherche effectuée par les musées, la perspective performative pose surtout la question du pouvoir de la validation et des moyens pris pour l'articuler et l'animer.

Cependant, la performativité présuppose que l'existence du récit soit garantie en tout temps. Contrairement au structuralisme, elle implique certes des éléments non narratifs, un travail de construction sociale et culturelle de la réalité, une contextualisation importante et une dynamique entre ce contexte, le récit même et la réalité, mais la performativité du récit implique, précisément, un récit, aussi poreux ou instable soit-il. Or, un récit n'est pas nécessairement présent. Revenons sur la définition du récit selon la perspective performative, présentée plus haut: un récit constitue un assemblage perçu d'éléments non aléatoires. C'est cette perception qui, en somme, fait agir. Le problème, c'est que cette perception ne survient pas nécessairement dans tous les cas, ne survient pas nécessairement de manière contrôlée et ne survient pas nécessairement en totalité. J'ajouterais que ceci est particulièrement vrai lorsqu'il est question de traduction de recherches muséales en récits. En effet, la plupart du temps, le visiteur est relativement laissé à lui-même dans une exposition muséale; cette liberté fait en sorte que le récit constitué dans l'exposition n'advient pas nécessairement comme prévu $^{33}$. Ainsi, l'enjeu du pouvoir, si cher à la théorie performative, doit être réévalué, puisque l'impact d'une exposition peut être totalement différent de ce qui a été planifié à l'origine. À la lumière de cette limite de la perspective performative, comment considérer le récit, alors que ses frontières sont totalement éclatées?

32 CITÉ DE LA MUSIQUE. Exposition Europunk. 15 octobre $2013-19$ janvier 2014.
33 Ces remarques sont à mettre en parallèle avec les théories de la médiation et de la réception. Voir DAVALLON, Jean. « Réflexions sur la notion de médiation muséale ». L'Art contemporain et son exposition (1). Paris: L'Harmattan, 2002, p. 41-61. 


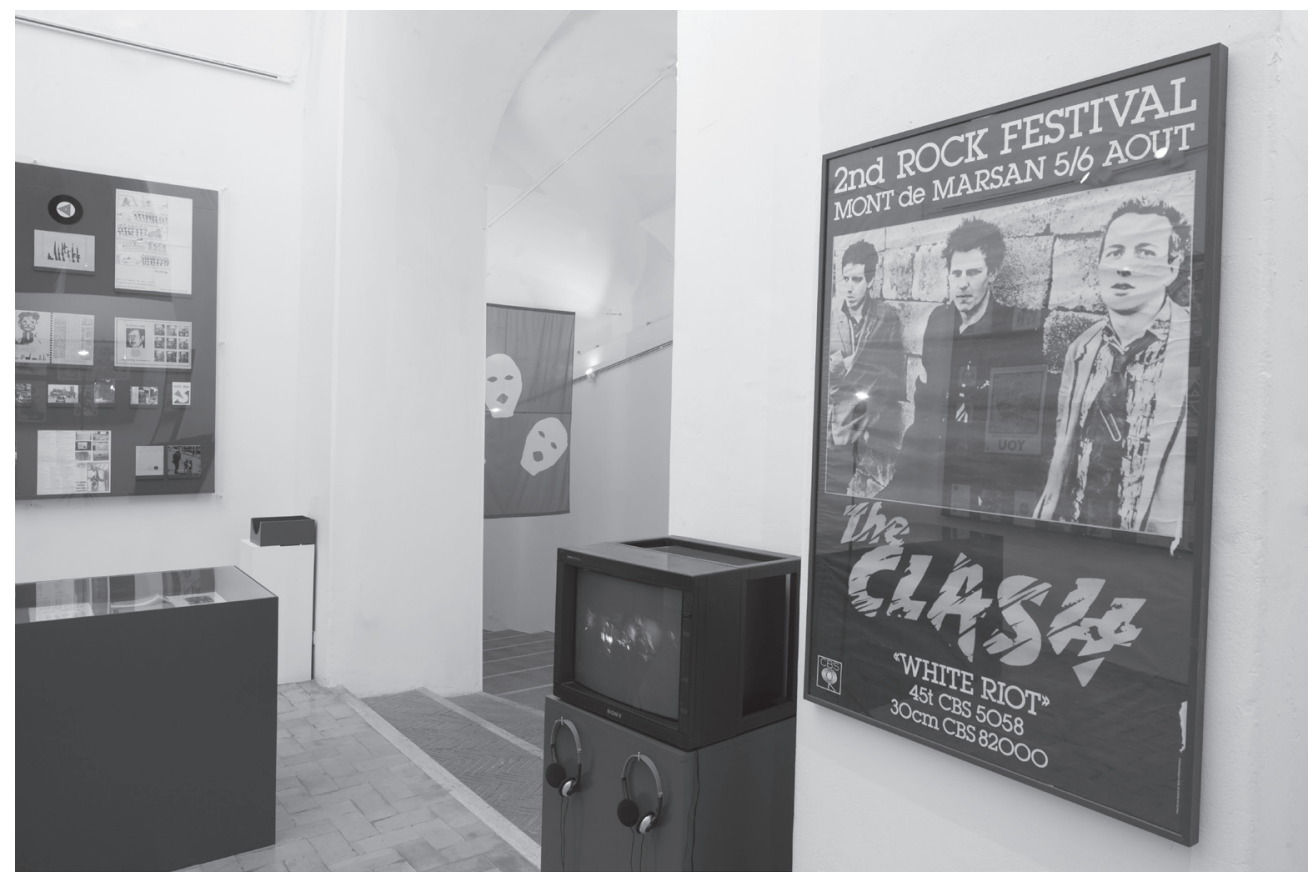

Europunk la culture visuelle punk en Europe, 1976-1980.

[Photo: Giovanni De Angelis]. (c) Giovanni De Angelis, photographe, Académie de France à Rome - Villa Médicis].

\section{Récits ontogénétiques}

Pour cet angle théorique, je m'inspire d'abord d'un article portant sur la cartographie. Même si le lien entre les cartes et les récits semble à première vue ténu, la perspective ontogénétique développée et expliquée par les auteurs, inspirée notamment par le penseur français Michel de Certeau ${ }^{34}$, peut être appliquée à d'autres formes culturelles, comme le récit: " we think it is a productive way to think about the world [...] It acknowledges how life unfolds in multifarious, contingent and relational ways ${ }^{35}$. » L'ontogénèse fait référence au développement de l'individu; en ce sens, la théorie ontogénétique concerne le développement, ou encore l'émergence, de phénomènes sociaux et culturels. Le récit, considéré de manière ontogénétique, est ainsi dénaturalisé. En fait, il ne faudrait pas parler de récit, mais plutôt de narration, soit l'acte constant de raconter ${ }^{36}$. L'enjeu n'est plus « ce qu'un récit est » ou « ce qu'un récit produit », mais « comment un récit devient ».

La narration émerge à travers un ensemble de pratiques, qui peuvent être d'ordres divers, comme je le démontre ci-dessous avec trois exemples. Les pratiques narratives peuvent se comparer aux tactiques telles que présentées par de Certeau, où les « consommateurs"

34 DE CERTEAU, Michel. L'invention du quotidien I: Arts de faire. Paris: Gallimard, 1990, p. 139-191.

35 KITCHIN, Rob et Martin DODGE. « Rethinking Maps ". Progress in Human Geography, vol. 31, n 3, 2007, p. 335-337.
36 D'ailleurs, pour le reste de l'article, pour être cohérente avec la théorie ontogénétique, j'utiliserai le concept de « narration " plutôt que celui de " récit ". 
doivent s'approprier les stratégies conçues par les producteurs ${ }^{37}$. Elles ne sont donc jamais totalement déterminées par ceux qui imaginent et confectionnent les récits ${ }^{38}$. De cette manière, les narrations se renouvellent constamment et s'actualisent toujours différemment; elles deviennent transitoires, éphémères et contingentes. Ainsi, même si I'information transmise dans un récit est techniquement la même pour tout le monde, l'actualisation en narration que chacun en fait peut être totalement différente d'une personne à l'autre. De plus, la narration s'adapte aux besoins du moment. Elle est donc un processus radical, poussée à un point extrême où les récits existent à peine, où ils sont plutôt en constante (re)naissance.

Pour illustrer les narrations ontogénétiques dans Europunk, je m’appuie sur trois exemples. Tout d'abord, un cas assez classique, facilement imaginable pour d'autres expositions muséales: visiter l'exposition à l'envers ou dans un ordre différent de celui implicitement imposé par la salle elle-même, par la disposition des artéfacts ou par les panneaux de textes. Un visiteur qui parcourrait Europunk dans le désordre regarderait les différentes sections de manière assez aléatoire. Ainsi, il pourrait commencer sa visite par la dernière section, "New Wave », et terminer par la section "Sex Pistols", qui se veut une introduction. Le visiteur composerait le récit de l'exposition, sa propre narration de celle-ci, au fur et à mesure de sa visite, assemblant les mots et les choses selon ses connaissances préalables, son intérêt et ses expériences. Cela ne diffère pas d'un autre visiteur qui parcourrait l'exposition dans l'ordre, à l'exception que la narration produite par les deux visiteurs serait différente; la narration est donc contingente.

Le deuxième exemple s'applique seulement à Europunk. En effet, un visiteur pourrait très bien se concentrer sur la double chronologie, expliquée précédemment, plutôt que sur les artéfacts des six sections. En étant absorbé par les événements sociaux, politiques et économiques de la décennie 1970, un visiteur pourrait très bien être amené à oublier l'objet principal de l'exposition, éclipsé dans et par la narration constituée par cette mise en contexte. De plus, cette double chronologie est dense et informative au point où il est impossible de tout retenir. Seulement une fraction de ces éléments peut être retenue pour construire la narration de l'exposition. En ce sens, la narration est éphémère.

Enfin, revenons sur la salle Play it, qui met des instruments de musique à la disposition des visiteurs. Imaginons le cas d'une jeune fille qui, dans le cadre d'une visite scolaire à Europunk, aurait limité sa visite à cet atelier, sans s'attarder aux artéfacts, aux textes ou à la double chronologie. Cette adolescente rêve de jouer de la musique depuis longtemps, mais elle n'avait jamais pu essayer un instrument. Play it lui

37 DE CERTEAU, L'invention du quotidien I, op. cit., p. $50-68$.

38 Je pense ici par exemple à un récit qui serait "préformé " pour les récepteurs, comme cela est le cas dans un musée (mais aussi les bulletins d'information, des articles de journaux, des articles techniques et scientifiques - comme celui que vous êtes en train de lire). Évidemment, plusieurs récits ne proviennent pas d'une source officielle (les rêves durant le sommeil, une chicane entre conjoints, une recette improvisée lors d'un souper), et l'aspect continuellement fabriqué de ces récits est encore plus évident. 
offre la possibilité d'en essayer quelques-uns. Ainsi, la narration qu'elle en développe est centrée autour de sa nouvelle expérience, ses sensations, ses observations, ses émotions. Peut-être aimera-t-elle tellement cette narration qu'elle épargnera suffisamment pour acheter une guitare basse ou une batterie? La narration d'Europunk apparaît donc comme transitoire.

Évidemment, la perspective ontogénétique suppose que chaque visiteur se raconte différemment l'exposition. En fait, non seulement cette extrême contingence est-elle à remarquer, mais la narration s'effectue continuellement, de manière fuyante, instable et furtive, en fonction de plusieurs conditions qui ne sont pas nécessairement reliées aux artéfacts, aux textes et à la disposition de ceux-ci. Cette précarité radicale fait en sorte qu'il peut paraître vain et péremptoire d'appliquer cette théorie en vue d'une traduction de recherches muséales en récits. Que faire lorsque tout récit devient narration évanescente chez les visiteurs? Comment savoir ce que les visiteurs retiennent de l'exposition, si la narration n'est jamais la même?

En guise de conclusion, je souhaite aborder la notion de musée participatif, qui se marie bien avec la perspective ontogénétique. Cette notion se centre justement autour du visiteur, de l'implication que celui-ci peut déployer dans un musée. Une des prémisses de base est de faire confiance aux habiletés créatrices des visiteurs, puisque le musée participatif encourage une appropriation des contenus ${ }^{39}$. Ainsi, l'expression des diverses narrations ontogénétiques des visiteurs peut faire partie de stratégies participatives mises en place par un musée pour une exposition (dans le cadre de visites guidées, de journées d'études, d'ateliers, des commentaires sur le site Web du musée, et bien d'autres). Les visiteurs peuvent même " contribuer » aux recherches effectuées par les musées. Surtout que les outils pour permettre de telles contributions se font dorénavant légion.

Je fais référence, bien entendu, aux réseaux sociaux comme Facebook, Twitter, Instagram ou YouTube. En cette décennie où Internet est devenu à maints égards synonyme de tels réseaux, il est difficile de passer à côté de ces outils participatifs. Selon Martin Lister et ses collègues, l'interactivité se définit comme " a more powerful sense of user engagement with media texts, a more independent relation to sources of knowledge, individualised media use, and greater user choice ${ }^{40} "$, mais la plupart du temps le terme réfère à toute la palette des diverses interventions disponibles à l'usager face à un contenu médiatique. Les liens à faire avec le musée participatif sont très nombreux: les musées peuvent demander à un groupe d'internautes de participer à une exposition en soumettant des photos sur Instagram, ils peuvent

39 THE PARTICIPATORY MUSEUM. Preface: Why Participate? <http://www.participatorymuseum.org/preface/> (consulté le 2 novembre).
40 LISTER, Martin, Jon DOVEY, Seth GIDDINGS, Iain GRANT et Kieran KELLY. New Media: A Critical Introduction. Londres et New York: Routledge, 2009, p. 21. 
organiser un concours de création de mèmes ${ }^{41}$ relatifs à une exposition, ils peuvent questionner leurs fans Facebook pour savoir ce qu'ils ont retenu d'une exposition particulière. Ce dernier exemple pourrait également permettre aux musées d'effectuer un genre de monitoring pour connaître les différentes narrations ontogénétiques produites par les visiteurs. Dans Europunk, cependant, pour des raisons de droits d'auteurs, les visiteurs ne pouvaient pas prendre de photos avec les œuvres présentées, coupant ainsi le Musée de la musique d'une possibilité d'interactivité ${ }^{42}$ qui aurait pu lui être bénéfique.

D'ailleurs, la créativité - mot bien à la mode en ce moment! - est un bon moyen de cadrer l'interactivité. Par exemple, une initiative comme Museomix ${ }^{43}$, un marathon créatif connecté où des professionnels de plusieurs domaines font éclater les frontières habituelles du musée en proposant des projets reliés à des expositions, favorise la créativité non seulement chez les muséologues (qui doivent réaliser ces projets en trois jours seulement), mais aussi chez les visiteurs qui testent ces projets. Justement, ces projets sont interactifs, au sens où ils incluent la participation des visiteurs, sous une forme ludique ou avec l'aide de technologies, ou encore en prenant des récits d'exposition comme base pour la participation. Cette impulsion créative des musées non seulement changera à coup sûr les narrations ontogénétiques des visiteurs, mais constitue peut-être le catalyseur vers une plus grande place pour la théorie ontogénétique dans les musées.

41 Un mème Internet est une idée complexe réduite sous la forme d'une unité distincte et mémorable et qui circule massivement sur le Web. Voir : KNOW YOUR MEME. < http:// knowyourmeme.com/> (consulté le 2 novembre 2014).
42 Par ailleurs, l'interactivité était, à une époque sans le Web commercial, un des moteurs du mouvement punk. 43 Voir MUSEOMIX. <http://www.museomix.org/> (consulté le 7 novembre 2014). 